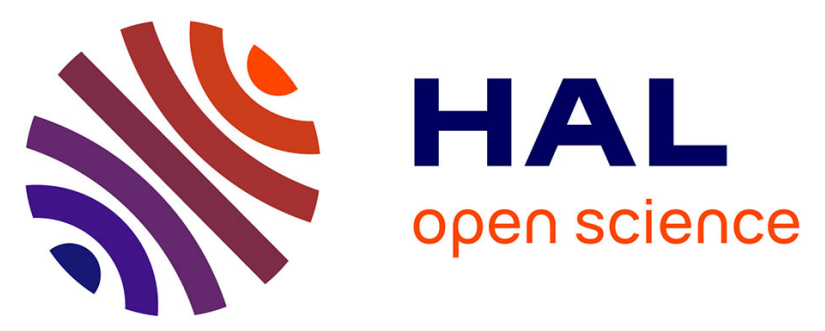

\title{
Predistortion system implementation based on analog Neural Networks for linearizing High Power Amplifiers transfer characteristics
}

Blaise Mulliez, Emmanuel R. Moutaye, Hélène Tap, Laurent Gatet, Francis Girard

\section{To cite this version:}

Blaise Mulliez, Emmanuel R. Moutaye, Hélène Tap, Laurent Gatet, Francis Girard. Predistortion system implementation based on analog Neural Networks for linearizing High Power Amplifiers transfer characteristics. Telecommunications and Signal Processing (TSP), 2013 36th International Conference on, Jul 2013, Rome, Italy. pp.412-416, 10.1109/TSP.2013.6613964 . hal-00942355

\section{HAL Id: hal-00942355 \\ https://hal.science/hal-00942355}

Submitted on 27 May 2014

HAL is a multi-disciplinary open access archive for the deposit and dissemination of scientific research documents, whether they are published or not. The documents may come from teaching and research institutions in France or abroad, or from public or private research centers.
L'archive ouverte pluridisciplinaire HAL, est destinée au dépôt et à la diffusion de documents scientifiques de niveau recherche, publiés ou non, émanant des établissements d'enseignement et de recherche français ou étrangers, des laboratoires publics ou privés. 


\title{
Predistortion System Implementation Based On Analog Neural Networks For Linearizing High Power Amplifiers Transfer Characteristics
}

\author{
B. Mulliez ${ }^{1,2,3}$, E. Moutaye ${ }^{1,2}$, H. Tap ${ }^{2,4}$, L.Gatet ${ }^{1}$, F. Gizard $^{1}$
}

\begin{abstract}
In order to correct non-linearities due to High Power Amplifiers (HPA) operating near saturation in telecommunication transceivers, a new adaptive predistortion system based on analog Neural Networks (NNs) was developed. Based on size, consumption and bandwidth considerations, Multi-Layer Perceptron (MLP) type NNs were implemented in a $0.6 \mu \mathrm{m}$ CMOS ASIC. The NNs parameters are digitally updated with a computer, depending on simulation conditions (temperature drifts, ageing variations). The interface between the analog part and the software updating system is integrated in an analogdigital PCB including a FPGA, 6 analog-to-digital converters and 62 digital-to-analog converters. This paper describes the realization of each part of the breadboard system and presents experimental validation results of the whole predistortion module.
\end{abstract}

Index Terms-High Power Amplifiers, Neural Network Hardware, Predistorsion, Spatial Telecommunications.

\section{INTRODUCTION}

In satellite telecommunications, power efficiency and spectral efficiency are prominent concerns. For power efficiency purposes, High Power Amplifiers (HPA), such as Travelling Wave Tubes Amplifiers (TWTA) or Solid State Power Amplifiers (SSPA), are operating close to saturation, so as to get the maximum power efficiency onboard the satellite. Nevertheless, this leads to amplitude and phase non-linearities and the link transmission quality is deteriorated [1]. The combination of spectrally efficient modulations with non-constant envelope together with non-linearities leads to strong distortions of the transmitted signals.

There are several solutions to operate the HPA close to its saturation point without generating non-linearities [2]-[6]. One is to implement a module directly onboard the payload, just before the HPA, in order to obtain a linear transfer characteristic at the HPA output [4]-[6]. This solution, called the predistortion method, has the advantage to correct the HPA non-linearities directly onboard the satellite. It is also highly interesting because new satellite generations will have regenerative payloads. Therefore, the signals will be available in baseband, before modulation. Supposing that the frequency transposition does not distort the signal envelope, it is possible to apply a predistortion on the baseband signal before

1 CNES, 18, avenue Edouard Belin, F-31401 Toulouse Cedex 9, France

2 CNRS-LAAS, 2, rue Camichel, F-31071 Toulouse Cedex 7, France

3 DGA/DS-MRIS, 7, rue des Mathurins, F-92221 Bagneux, France

${ }^{4}$ Univ. de Toulouse, INP, F-31400 Toulouse, France

The authors would like to thank all the Hyperfrequency team of CNES for their helpful contributions and the French General Armament Direction (DGA) for their financial support. frequency transposition and HPA amplification. Thus, the predistortion system may operate in lower frequency bands, which opens new technological solutions never explored before.

Another point to have in mind is that the HPAs integrated onboard satellites undergo small amplitude and phase variations due to temperature drifts, ageing, and so on. Thus, if the predistortion module is integrated in the satellite payload, it must be adaptive and modify the predistortion transfer characteristic as a function of the drifts.

This paper presents a predistortion module developed in order to take into account all the concerns presented above. The different choices made from the module definition up to the breadboard integration are summed up and justified in the next parts: the way to model the HPA and the linearizer transfer function are developed in part II. Then, parts III, IV and V detail respectively the integration of the analog predistortion system, the instrumentation of the analog to digital interface between the analog part and the updating software, and the experimental tests achieved to confirm the $\mathrm{NN}$-based predistortion system ability to linearize the different HPA transfer characteristics.

\section{MOdELING AND LINEARIZATION OF POWER AMPLIFIERS}

\section{A. Modeling of HPA transfer characteristic}

Since the HPA transfer characteristic has to be linearized directly onboard the satellite, the HPA experimental behavior has to be modeled. For this, it is necessary to collect experimental signal data in relation with its functioning mode at regular time intervals and to analyze these data in order to operate the adequate processing.

Among the existing method to model HPA (Saleh [7], polynomial [8], Volterra [9]), a trade-off between precision and complexity of the model has to be determined. As a matter of fact, the better the precision of the model is, the heavier the computational workload is. Table I presents the different types of models found in the literature with their advantages and drawbacks. The memory effects modeling shown in the second column may be defined as the dependence of the distortion on the input signal frequency variation. These effects, hard to model, have not been taken into account in this study, so the HPA is considered as memoryless.

The memoryless Saleh model was chosen to describe a $80 \mathrm{~W}$ Ka-Band TWTA developed during a French Space Agency (CNES) research program. This model has the advantage 
TABLE I

COMPARISON BETWEEN DIFFERENT HPA MODELS

\begin{tabular}{c|c|c|c}
\hline \hline Model & Memory effect & Precision & Complexity \\
\hline Saleh & No & Not precise & Very simple \\
Memoryless polynomial & No & Not precise & Simple \\
Volterra & Yes & Very precise & Very complex \\
Polynomial with memory & Yes & Precise & Complex \\
Hammerstein & Yes & Precise & Complex \\
Wiener & Yes & Precise & Complex \\
Hammerstein-Wiener & Yes & Precise & Very complex \\
Wiener parallel & Yes & Precise & Complex \\
Neural Network & Yes & Very precise & Complex \\
\hline \hline
\end{tabular}

to be quite simple to use. Moreover, it fits well the HPA characteristic at first order. Furthermore, by varying the scalar parameters of the model, it is possible to model variations of the amplifier characteristics. The scalar parameters' values of this model were provided by CNES and allow modeling with satisfying precision the transfer characteristic of the HPA. Fig. 1 shows a representation of the normalized output amplitude and of the output phase shift as a function of the input amplitude up to the saturation point. Fig. 2 represents different HPA transfer characteristics issued from the model in amplitude and phase.

\section{B. Linearization of power amplifiers}

The linearization of a HPA consists in reducing its nonlinearities to optimize the efficiency, the amplifier output

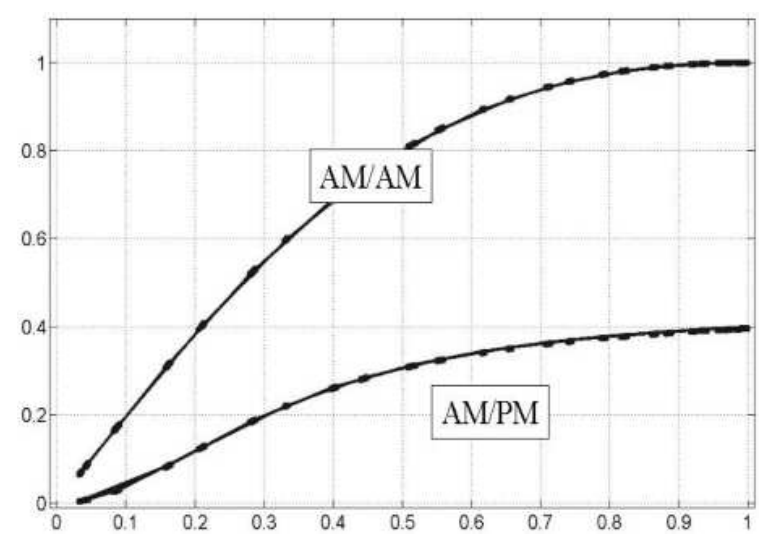

Fig. 1. Representation of normalized AM/AM conversion and AM/PM conversion as a function of the normalized input amplitude

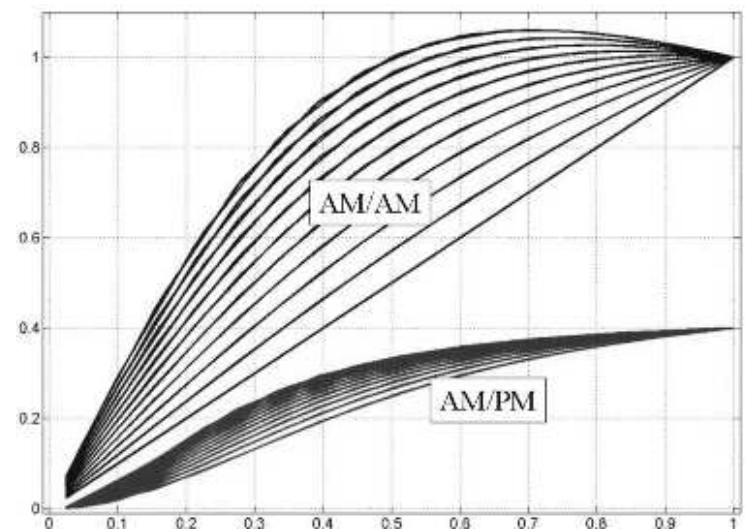

Fig. 2. Normalized HPA transfer characteristics in amplitude (V) and phaseshift (radians) as a function of the normalized input amplitude (V) for different Saleh scalar values power and the signal integrity. Several techniques are referenced in the literature in order to proceed with predistortion [4]-[6], [10], [11]. The technique described in [4] is particularly interesting since the output signal is used to monitor the predistortion module but does not directly participate in the linearization scheme (Fig. 3).

The AM/AM and the AM/PM transfer characteristics of the predistortion functions are deduced from the HPA ones. By comparison with others non-linear modeling systems, the predistortion with Neural Networks (NNs) has the advantage of a high inherent parallelism with a simple repetitive scheme, which makes them attractive for integrated circuit technologies implementation purposes [12]-[13]. Moreover, NNs have the ability to be adaptative in that their parameters may be updated. In order to predistort continuously baseband $(I, Q)$ input signals, the predistortion system of this study was developed and achieved with Multi-Layer Perceptrons (MLP) NNs [14], [15], whose structure is developed in part III. The architecture of the predistortion system was developed in [16] and is presented in Fig. 4.

\section{ANALOG PREDISTORTION SYSTEM}

An analog implementation was chosen for the NN rather than a digital one, so as to optimize the circuit bandwidth, the input signals' dynamics and the power consumption [12]. The digital updating system was implemented in a FPGA, in order to be more efficient and easily reconfigurable.

The inner structures of the analog predistortion module and of the MLP-type NNs were described in [16] and are shown respectively in Fig. 5 and Fig. 6.

Computer simulations confirmed the chosen structure ability to linearize HPA gain distortion, for varying simulation conditions and by taking into account variations due to technology non-homogeneities and voltage offsets together with

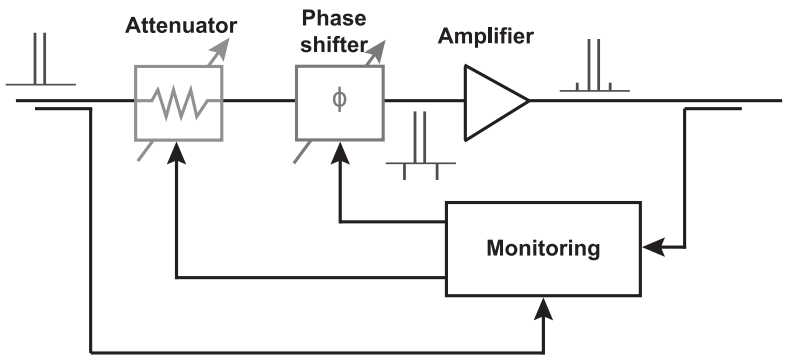

Fig. 3. Architecture of the predistortion feedback technique

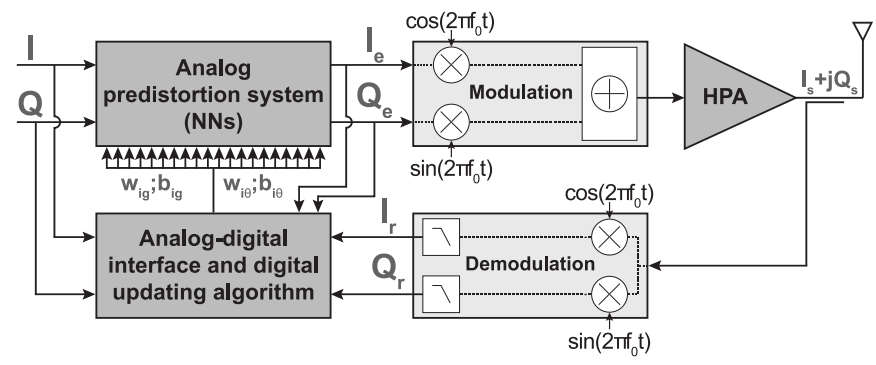

Fig. 4. Global predistortion system architecture 


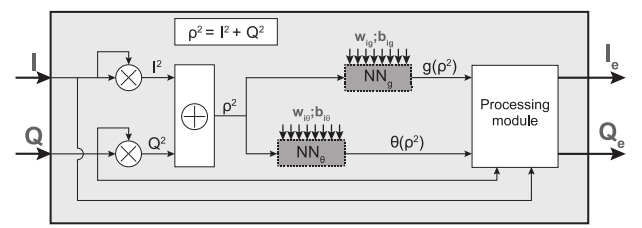

Fig. 5. Schematic of the analog predistortion module

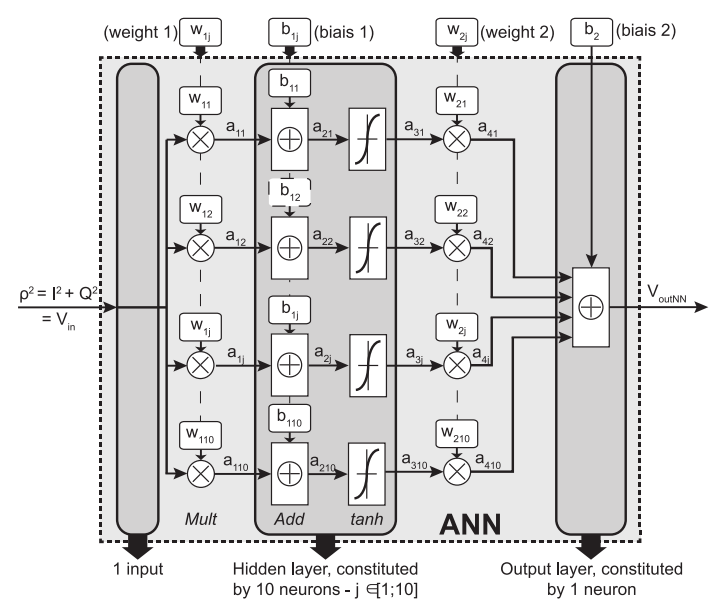

Fig. 6. Multi-Layer Perceptron Neural Network structure

corruption of the NN signals by white noises [14], [15]. The feedforward NNs were integrated in CMOS $0.6 \mu \mathrm{m}$ technology. The NN circuits are detailed in [17], [18].

In order to correct both non-linearities represented in Fig. 1, the predistortion output signals $I_{e}$ and $Q_{e}$ have to follow:

$$
\begin{aligned}
& I_{e}=I \cdot g\left(\rho^{2}\right) \cos \left(\theta\left(\rho^{2}\right)\right)-Q \cdot g\left(\rho^{2}\right) \sin \left(\theta\left(\rho^{2}\right)\right) \\
& Q_{e}=Q \cdot g\left(\rho^{2}\right) \cos \left(\theta\left(\rho^{2}\right)\right)-I \cdot g\left(\rho^{2}\right) \sin \left(\theta\left(\rho^{2}\right)\right)
\end{aligned}
$$

with $\rho^{2}$ the square modulus of signals $I$ and $Q$, and $g\left(\rho^{2}\right)$ and $\theta\left(\rho^{2}\right)$ the respective outputs of $\mathrm{NNg}$ and $\mathrm{NN} \theta$ [3]. The functions $g$ and $\theta$ may be modified as a function of the HPA drifts by updating the $31 \mathrm{NNs}$ parameters of each NN after their calculation by the algorithmic part. As the AM/PM distortion amplitude is lower than 0.4 radian, (1) and (2) may be approximated by:

$$
\begin{aligned}
I_{e} & =I \cdot g\left(\rho^{2}\right) \cdot\left(1-\frac{\theta\left(\rho^{2}\right)}{2}\right)-Q \cdot g\left(\rho^{2} \theta\left(\rho^{2}\right)\right. \\
Q_{e} & =Q \cdot g\left(\rho^{2}\right) \cdot\left(1-\frac{\theta\left(\rho^{2}\right)}{2}\right)-I \cdot g\left(\rho^{2}\right) \theta\left(\rho^{2}\right)
\end{aligned}
$$

The processing part, achieved by the analog predistortion system (Fig. 5), realizes both functions (3) and (4) [16].

Besides, to ensure that the linearizer does not inject signals above the input saturation voltage of the HPA, a limiter may be used between the predistortion module and the amplifier.

\section{UPDATING SYSTEM}

\section{A. Structure}

It is important to perfectly control the variations of the predistorted signals $\left(I_{e}, Q_{e}\right)$ as a function of the input signals $(I, Q)$ and the parameters of each NN. That is why the 6 signals $I, Q, I_{e}, Q_{e}, I_{r}$ and $Q_{r}$ issued from the analog predistortion system are sampled. So, in order to control the whole system linearity, the demodulated HPA output signals $\left(I_{r}, Q_{r}\right)$ are compared to the input signals $(I, Q)$ in order to observe if both couples of signals are proportional and phased. If so, the NNs' parameters are not updated. If not, a software training phase begins with the stored raw data, in order to calculate the new NNs' parameters, and then better linearize the distorted HPA transfer characteristic. Once the 62 NNs' parameters updated, they are converted and injected into the analog integrated NNs via two ICs of 32 DACs. The training procedure of the NNs is described in part V-B. Fig. 7 represents the structure of the analog-digital interface between the analog predistortion system and the computer developed in [16] and [18].

\section{B. Realization}

Fig. 8 represents a photograph of the whole analog-digital interface. The 6 analog signals to sample are injected at (1). After the analog-to-digital conversions, the data are stored in the FPGA (2) before being transmitted to the computer via the RS232 link (3).

After calculation, the updated NNs' parameters are sent to the FPGA via the RS232 link. If necessary, the 62 digital parameters are converted by 62 DACs distributed in two ICs of 32 DACs (4) in order to be injected at the same time in the analog predistortion system.

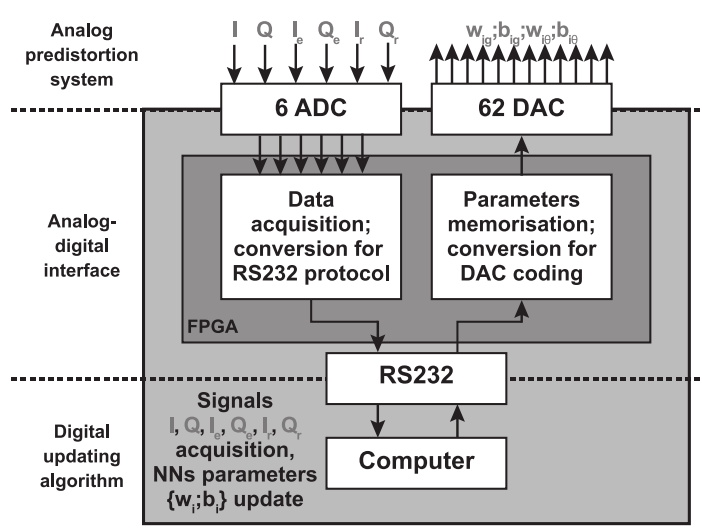

Fig. 7. Analog-digital interface

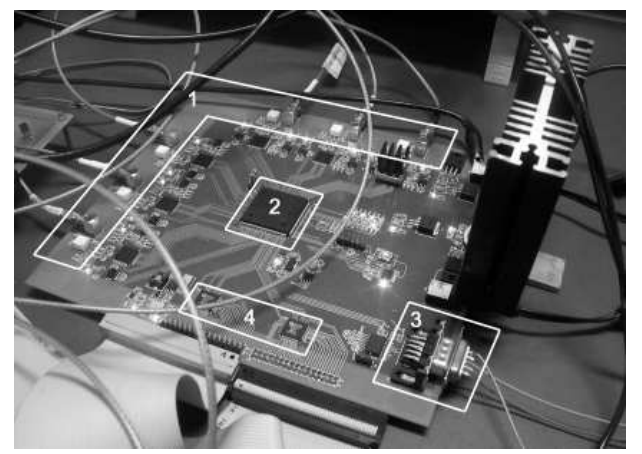

Fig. 8. Analog-digital interface photograph 


\section{EXPERIMENTAL TESTS ACHIEVED ON THE GLOBAL PREDISTORTION SYSTEM}

\section{A. Preliminary setup}

Every elementary function of the NN (adder, multiplier) may present an undesired offset or multiplying factor at its output. Before determining the linearization ability of the system, these errors have to be characterized. Therefore, a preliminary setup algorithm was performed in order to determine these errors. For each $\mathrm{NN}$ and for each of the 31 elementary functions, the imprecision was accurately modeled and memorized. The training software was then adapted with the adequate constants.

\section{B. Test procedure}

An interface was developed using the NI LabWindows ${ }^{\mathrm{TM}} / \mathrm{CVI}$ environment to control the analogdigital circuit and process the input and output data.

The test procedure applied to determine the linearization ability of the whole predistortion system is detailed in Fig. 9.

First, the user has to specify the Saleh model which fits better to the HPA characteristics to linearize. Then, the user defines the amplitude and phase error criterions for which NNs' parameters have to be updated. Then, a sequence of $I$ and $Q$ voltages is performed and injected into the linearizer. The waveforms' frequency relative to $I$ and $Q$ signals may vary arbitrary between $0.1 \mathrm{MHz}$ and $25 \mathrm{MHz}$.

A first acquisition of $I, Q, I_{e}$ and $Q_{e}$ is achieved for null weights and biases. After that, $I_{r}$ and $Q_{r}$ voltages are determined as a function of $I_{e}$ and $Q_{e}$ sampled values and Saleh's scalars. Then, $(I, Q),\left(I_{e}, Q_{e}\right)$ and $\left(I_{r}, Q_{r}\right)$ are compared in order to achieve an intercorrelation and synchronize the signals on the same sample.

After the intercorrelation step, $(I, Q)$, and $\left(I_{r}, Q_{r}\right)$ are compared in amplitude and in phase. If the errors are lower than the criterions defined by the users, the NNs' parameters

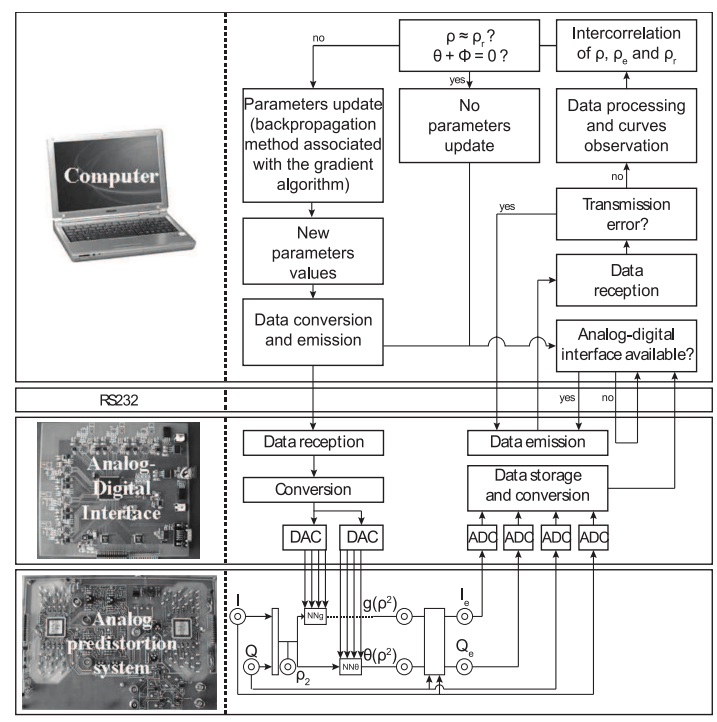

Fig. 9. Test procedure to validate the linearization ability of the predistortion system are not modified. If not, both $\mathrm{NNs}$ are trained in order to respect both criterions. The backpropagation method, associated with the simple gradient algorithm [19], is used on relevant data. As soon as the convergence is observed and the user's criterions respected, the algorithm stops, and the updated NNs' parameters are validated with all the acquisition data. After the validation phase, the NNs' parameters are transmitted to the analog predistortion system. Then, a new acquisition is automatically achieved to confirm that the new parameters' values experimentally correct both AM/AM and AM/PM nonlinearities.

\section{NN convergence}

The developed NI LabWindows ${ }^{\mathrm{TM}} / \mathrm{CVI}$ code allows the user to choose between three linearization criterions: the user may specify the maximum tolerable error between $(I, Q)$ and $\left(I_{r}, Q_{r}\right)$, or the maximum average tolerable error, or a fixed number of iterations of the backpropagation algorithm. In any case, an absolute maximum number of iterations was fixed to 100000 in order to avoid linearization system divergence. Nonetheless, even with 100000 iterations, the processing time remains under one second.

\section{Experimental results}

The first experimental tests were achieved with the Saleh's scalars provided by the French Space Agency (CNES). These parameters correspond to the transfer characteristic of a specific HPA in well-defined experimental conditions. After having observed $(I, Q)$ and $\left(I_{r}, Q_{r}\right)$ sampled data, the AM/AM and $\mathrm{AM} / \mathrm{PM}$ responses were plotted in order to validate that both couples of signals are quite identical.

Fig. 10 presents an example of experimental AM/AM transfer characteristics observed after transposition of the HPA output, respectively without predistortion (solid curve), with an ideal predistortion system (solid straight) and with the implemented predistortion system (dots). With null parameters before the beginning of the test procedure, the dots are superposed with the solid curve (Fig. 1). The predistortion system is able to correct both non-linearities in less than one second with a signal-to-error ration (SER) higher than $30 \mathrm{~dB}$. This can be interpreted as amplitude and phase mean relative errors lower than $5 \%$.

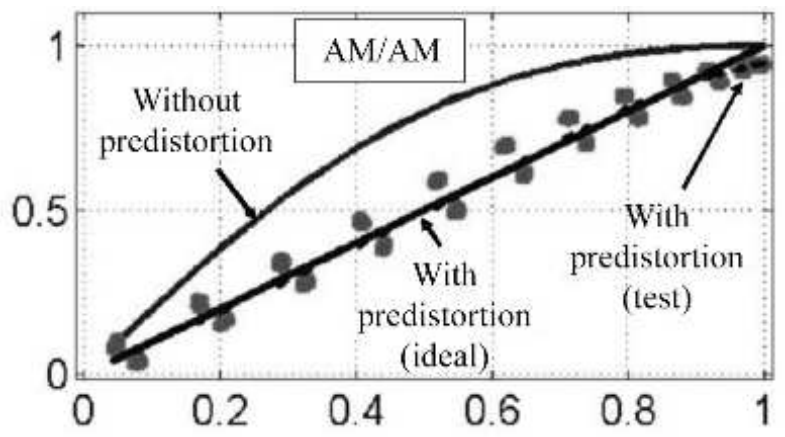

Fig. 10. Representation of the normalized experimental AM/AM curves without predistortion (solid curve) and with predistortion (solid straight: ideal; dots: experimental points) as a function of the normalized input amplitude 
Then, the Saleh's scalars were modified to prove that the predistortion system is able to correct HPA transfer characteristic drifts due to temperature variation, ageing, and so on, or to linearize other HPAs whose transfer characteristics fit with these Saleh model scalar values. The different transfer characteristics tested are represented in Fig. 2. In each case, the predistortion system is able to correct both AM/AM and AM/PM non-linearities with an experimental SER higher than $30 \mathrm{~dB}$. This result observed with arbitrary analog waveforms belonging to the $[0.1 \mathrm{MHz} ; 25 \mathrm{MHz}]$ frequency spectrum makes us confident on the fact that this linearization method could be achievable for a digital modulation with a $25 \mathrm{MHz}$ bandwidth needed for a classical 16-QAM modulation at $100 \mathrm{Mbp} / \mathrm{s}$ [14], [15].

\section{CONCLUSION AND PERSPECTIVES}

The developed analog predistortion system was validated by correcting many well-defined distortion characteristics in laboratory conditions. Therefore, the analog predistortion system is able to linearize different HPAs whose transfer function fits with the Saleh model. Moreover, the satisfactory operation of the NNs' parameters' update instrumentation was checked by continuously changing the Saleh's coefficients. Thus, this project shows that it is possible to linearize HPAs with an integrated predistortion system based on analog neural networks.

The next step is the test of the whole structure with different HPAs, in varying experimental conditions. The test procedure will be more complex, since it requires expensive test benches and to properly adapt the linearizer breadboard in these environmental conditions. If these tests are relevant, the feasibility of adaptive HPA linearization with analog neural networks will be proved.

A new optimized version of the complete analog part will be integrated in an ASIC with a more recent technology in order to optimize bandwidth and power consumption, and to transform this laboratory breadboard into an engineering model. As for the digital part of the system, the processing part will be integrated in a microprocessor or a FPGA [20], and the interface between the analog and the digital parts will be updated with regard to the new available components.

Eventually, the processing part of the future optimized version will have to take into account the memory effects of the power amplifier, in order to allow linearization over larger bandwidths.

\section{REFERENCES}

[1] J. B. Sombrin, "Optimisation criteria for power amplifiers", in International Journal of Microwave and Wireless Technologies, 2011.

[2] S. Bouchired, M. Ibnkahla, D. Roviras, F. Castanié, "Equalization of satellite mobile channels with neural network techniques", in Space Communications 15, IOS Press, pp. 209-220, 1998-99.

[3] S. Bouchired, M. Ibnkahla, D. Roviras, F. Castanié, "Neural Networks : A Tool for Satellite UMTS Channel Equalization", in Proceedings of DSP 98, Nordwick, The Netherlands, Sept. 1998.

[4] F. Langlet, H. Abdulkader, D. Roviras, A. Mallet, F. Castanié, "Adaptive predistortion for Solid State Power Amplifier using Multi-Layer Perceptron", in Proceedings of Globcom 2001, pp. 325-329, San Antonio, Texas, USA, Nov. 2001.
[5] F. Langlet, H. Abdulkader, D. Roviras, A. Mallet, F. Castanié, "Comparison of Neural Network Adaptative predistortion Techniques for Satellite Down Links", in Proceedings of IJCNN 2001, pp. 709-714, Washington DC, USA, July 2001.

[6] G. Lazzarin, S. Pupolin, A. Sarti, "Nonlinearity Compensation in Digital Radio Systems", in IEEE Transactions on Communications, Vol. 42, No. 2/3/4, pp. 989-999, Feb./March/April 1994.

[7] A. Saleh, "Frequency-independent and frequency-dependant non linear models of TWT amplifiers", in IEEE Transactions on Communications, Vol. COMM 29, No. 11, pp. 1715-1720, Nov. 1981.

[8] S. Yi, S. Nam, S. Oh, J. Han, "Prediction of a CDMA output spectrum based on intermodulation products of two-tone test", in IEEE Transactions on Microwave Theory and Techniques, Vol. 49, No. 5, pp. 938946 , May 2001.

[9] V. Volterra, "Theory of Functionals and of Integral of IntegroDifferential Equations", in Dover Publications, Inc, N.Y., 1958.

[10] Y.Yang, and al., "New Linearization Method for the Modulated Signals with High Peak-to-Average Ratio : Peak-to-Average Ratio Reduction and Expension", in IEEE MTT-S Digest, pp. 777-780, 2002.

[11] H. M. Park, and al., "A New Predistortion Linearizer Using EnvelopeFeedback Technique for PCS High Power Amplifier Application”, in IEEE Radio and Wireless Conference, pp. 223-226, Aug. 1999.

[12] B. Beliczynski, "Incremental approximation by one-hidden-layer neural networks: discrete functions rapprochement", in Proceedings of the IEEE International Symposium on Industrial Electronics (ISIE '96), Vol. 1, pp. 392-397, 17-20 June 1996.

[13] S. Haykin, "Neural Network : A Comprehensive Foundation", Prentice Hall, 1994.

[14] L. Gatet, H. Tap-Béteille, D. Roviras, F. Gizard, "Integrated CMOS analog neural network ability to linearise the distorted characteristic of HPA embedded in satellites", in 4th IEEE International Workshop on Electronic Design, Test and Applications (DELTA 2008), Hong Kong, pp. 502-505, Jan. 23-25, 2008.

[15] D. Roviras, H. Abdulkader, H. Tap-Béteille, F. Castanié, M. Lescure, A. Mallet, "Multi-Layer Perceptron Neural Network Implementation and Integration in CMOS Technology", in International Conference on Information and Communications Technologies : From Theory to Application (ICCTA 94), Damascus, Syria, pp. 19-23, April 2004.

[16] L. Gatet, F. Bony, H. Tap-Béteille, E. Moutaye, F. Jayat, D. Roviras, F. Gizard, "Embedded updating system based on integrated NNs in order to achieve adaptative predistortion of non-linear HPA characteristics", in 26th IEEE International Instrumentation and Measurement Technology Conference (I ${ }^{2}$ MTC 2009), Singapore, May 5-7, 2009, pp. 838-841.

[17] L. Gatet, H. Tap-Béteille, M. Lescure, D. Roviras, A. Mallet, "Functional Tests of a 0.6 $\mu \mathrm{m}$ CMOS MLP Analog Neural Network for Fast on-board Signal Processing", in Analog Integrated Circuits and Signal Processing, Springer Science \& Business Media, Vol. 54, No. 3, pp. 219-227, March 2008.

[18] L. Gatet, H. Tap-Béteille, M. Lescure, "Design and Test of a CMOS MLP Analog Neural Network for Fast On-Board Signal Processin",in 13th IEEE International Conference on Electronics, Circuits and Systems, Nice, France, pp. 922-925, Dec. 2006.

[19] L. Gatet, H. Tap-Béteille, M. Lescure, "Real-Time Surface Discrimination Using an Analog Neural Network Implemented in a Phase-Shift Laser Rangefinder", in IEEE Sensors Journal, Vol. 7, No. 10, pp. 13811387, Oct. 2007.

[20] A. Uncini, L. Vecci, P. Campolucci, F. Piazza, "Complex-Valued Neural Networks with Adaptive Spline Activation Function for Digital Radio Links Nonlinear Equalization", in IEEE Trans. Signal Processing, Vol. 47, pp. 505-514, Feb 1999. 